Molecules 2005, 10, 383-393

molecules

ISSN 1420-3049

http://www.mdpi.org

\title{
Diastereoselective Synthesis of 2-Phenylselenenyl-1,3-anti-Diols and 2-Phenylselenenyl-1,3-anti-Azido-Alcohols via Hydroxy- and Azido-Selenenylation Reactions
}

\author{
Serena Riela, Carmela Aprile, Michelangelo Gruttadauria,* Paolo Lo Meo and Renato Noto \\ Dipartimento di Chimica Organica "E. Paternò", Università di Palermo, Viale delle Scienze, Parco \\ d’Orleans II, Pad. 17, 90128, Palermo, Italy. Tel. (+39) 091 596919, Fax (+39) 091596825. \\ * Author to whom correspondence should be addressed; e-mail mgrutt@unipa.it
}

Received 12 November 2004; in revised form: 18 November 2004 / Accepted: 19 November 2004 / Publsihed: 28 February 2005

\begin{abstract}
A method to synthesize 2-phenylselenenyl-1,3-anti-diols and 2-phenylselenenyl-1,3-anti-azidoalcohols via hydroxy- or azido-selenenylation of trans-allylic alcohols is reported. Moreover, the first example of hydroxyl-selenenylation of an allylic azide is presented. Yields ranging from moderate to good and diastereomeric ratios up to 95:5 are achieved.
\end{abstract}

Keywords: Azides, diols, seleniraniun ion.

\section{Introduction}

The 1,3-diol system is frequently found in the structure of biologically active natural products such as the macrolide antibiotics [1], and consequently, a wide variety of synthetic methods have been developed for these targets [2]. Moreover, chiral 1,3-aminoalcohol sequences are found in compounds of biological interest such as nucleoside antibiotics or in alkaloids. Indeed, several synthetic approaches to these moieties have been developed [3].

In the last years our group has been involved in the stereoselective synthesis of substitued oxygenated heterocyclic rings, such as tetrahydrofurans, tetrahydropyrans, $\delta$ - and $\gamma$-lactones, using electrophilic organoselenium reagents [4]. Mild reaction conditions and easy removal or subsequent functionalization of the phenylselenenyl residue are the major advantages of this chemistry [5].

Here we report a simple approach for the synthesis of open chain molecules such as 2phenylselenenyl-1,3-diols and 2-phenylselenenyl-1,3-azido-alcohols, as precursors of 1,3-diols and 
1,3-aminoalcohols. Recently we reported two examples of direct hydroxyl-selenenylation reactions of trans-allylic alcohols with good diastereoselectivity [4a]. This reaction has not been studied in detail [6]. We then exploited this reaction with other compounds together with the electrophilic azidoselenenylation reaction [7]. It has been reported that the addition of $\mathrm{PhSeN}_{3}$ to simple alkenes, as well as to activated alkenes, proceeds stereospecifically but not regiospecifically [8]. A radical process for azido-phenylselenenylation of double bonds has been also reported [9]. Recently, 2-phenylselenenyl1,3-syn-diols have been prepared by a cross-aldol reaction between benzaldehyde and $\beta$-phenylselenenylenoxysilanes followed by borane reduction [10]. Some 2-phenylselenenyl-1,3-syn-diols have been obtained by electrophilic addition of benzeneselenenic acid to allylic alcohols [11].

\section{Results and Discussion}

Compounds 1a-g were used as starting materials for our study.

\begin{tabular}{|c|c|c|c|c|c|}
\hline \multicolumn{6}{|c|}{$1 \mathrm{a}-\mathrm{g}$} \\
\hline 1 & $\mathbf{R}^{1}$ & $\mathbf{R}^{2}$ & 1 & $\mathbf{R}^{1}$ & $\mathbf{R}^{2}$ \\
\hline $\mathbf{a}$ & $\mathrm{Me}$ & $\mathrm{Ph}$ & $\mathbf{e}$ & $\mathrm{Ph}$ & $\mathrm{Ph}$ \\
\hline b & $i-\operatorname{Pr}$ & $\mathrm{Ph}$ & f & $\mathrm{EtOCOCH}_{2}$ & $\mathrm{Ph}$ \\
\hline c & $\mathrm{Me}$ & $p-\mathrm{Cl}-\mathrm{Ph}$ & g & $\mathrm{Me}$ & $\mathrm{CH}_{2} \mathrm{Ph}$ \\
\hline d & $\mathrm{Me}$ & $\mathrm{Me}$ & & & \\
\hline
\end{tabular}

\section{Scheme 1}

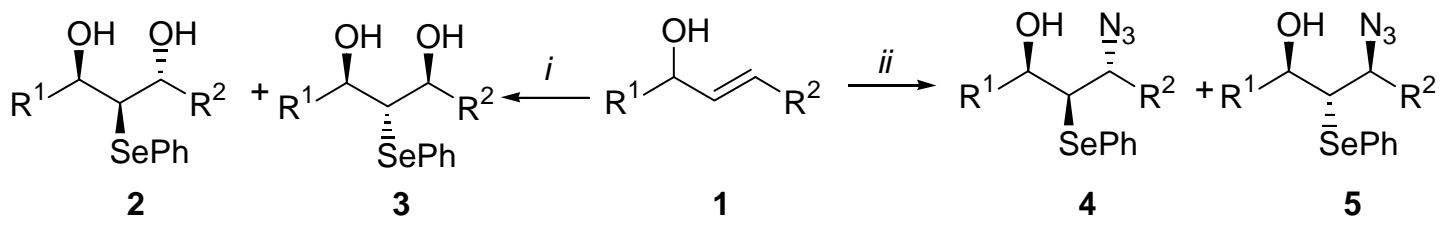

i: $\mathrm{PhSeCl}, \mathrm{CH}_{3} \mathrm{CN} / \mathrm{H}_{2} \mathrm{O}$, r.t.; ii: $\mathrm{PhSeX}$, solvent, $\mathrm{NaN}_{3}$.

First we studied the electrophilic hydroxy-selenenylation of alcohols 1a-f. We used for this purpose phenylselenenyl chloride in acetonitrile/water at room temperature. The intermediate seleniranium ion formed is attacked by the nucleophile, a water molecule, to give the diols $\mathbf{2}$ and $\mathbf{3}$. Yields ranged from moderate to good, with the less reactive compound being the alkyl substituted allylic alcohol 1d, whereas diastereomeric ratios were good, being at least 90:10. With $\mathrm{R}^{1}$ groups bigger than methyl, slight improvement in the ratios was observed.

Scheme 2

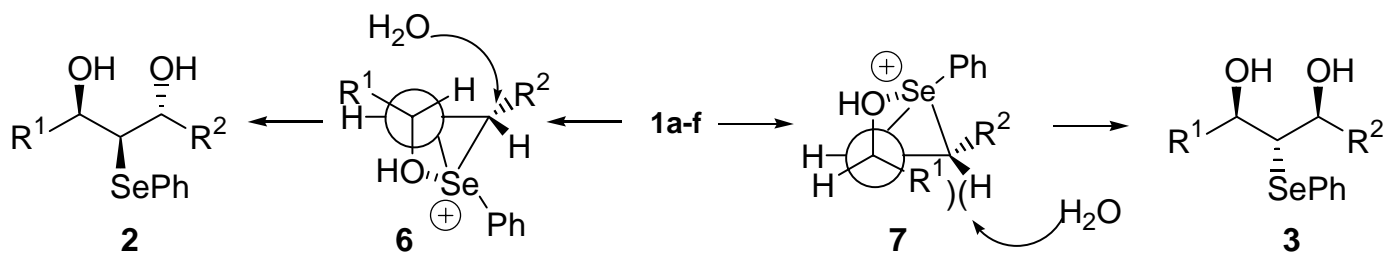


Table 1. Hydroxy-selenenylation of compounds 1a-f.

\begin{tabular}{lcccc}
\hline Entry & Compd. & $\begin{array}{c}\mathbf{2 + 3} \\
(\%)\end{array}$ & $\begin{array}{c}\mathbf{1} \\
(\%)\end{array}$ & $\mathbf{2 : 3}$ \\
\hline 1 & 1a & 88 & 11 & $90: 10$ \\
2 & 1b & 69 & 32 & $95: 5$ \\
3 & 1c & 63 & 24 & $92: 8$ \\
4 & 1d & 55 & 40 & $91: 9$ \\
5 & 1e & 77 & 11 & $94: 6$ \\
$6[4 \mathrm{a}]$ & 1f & 80 & $<5 \%$ & $95: 5$ \\
\hline
\end{tabular}

The stereochemistry of the major diastereoisomer was verified by the ${ }^{1} \mathrm{H}-\mathrm{NMR}$ spectra of compounds 2d-e and 3d-e. Indeed, the $\mathrm{CHSePh}$ signal in the major diastereoisomers 2d-e was a dd, while in the minor diastereoisomers 3d-e it was a triplet because of their symmetry. The stereochemical outcome of the reaction is depicted in the Scheme 2. Seleniranium ions $\mathbf{6}$ and $\mathbf{7}$ arise from the attack of the electrophilic $\mathrm{PhSeCl}$ on both sides of the carbon-carbon double bond. As a consequence of the stabilizing Se- $\mathrm{O}$ interaction, seleniranium ion $\mathbf{7}$ is less stable because of the steric interaction between the $\mathrm{R}^{1}$ group and hydrogen atom. In seleniranium ion $\mathbf{6}$ this interaction is absent.

Then we studied the electrophilic azido-selenenylation of alcohols 1. Preliminary investigations were carried out on compound 1a in order to find the best conditions. We considered different amounts of sodium azide, solvents and sources of electrophilic selenium reagent. Reactions were carried out for $24 \mathrm{~h}$ at room temperature. Results are reported in Table 2. Significant yield improvements were obtained using five equiv. of $\mathrm{NaN}_{3}$ instead of three equiv., however a further increase of the amount of $\mathrm{NaN}_{3}$ did not give improved yields but rather a less clean reaction (Table 2, entries 1-3). The use of other solvents such as dimethylformamide, acetonitrile and dimethoxyethane in place of dimethylsulfoxide gave poor yields (Table 2, entries 2,4-6). The use of the more reactive phenylselenenyl triflate gave better yields both in acetonitrile and dimethylsulfoxide, being, indeed, the latter the best conditions found (entries 7,8).

Table 2. Azido selenenylation of compound 1a.

\begin{tabular}{cccccc}
\hline Entry & PhSeX & Solvent & $\begin{array}{c}\mathbf{4 + 5} \\
\mathbf{( \% )}\end{array}$ & $\begin{array}{c}\mathbf{1} \\
\mathbf{( \% )}\end{array}$ & $\mathbf{4} / \mathbf{5}$ \\
\hline $1^{\mathrm{a}}$ & $\mathrm{PhSeCl}$ & $\mathrm{DMSO}$ & 50 & 8 & $86 / 14$ \\
$2^{\mathrm{b}}$ & $\mathrm{PhSeCl}$ & $\mathrm{DMSO}$ & 66 & 7 & $84 / 16$ \\
$3^{\mathrm{c}}$ & $\mathrm{PhSeCl}$ & $\mathrm{DMSO}$ & 46 & 18 & $85 / 15$ \\
$4^{\mathrm{b}}$ & $\mathrm{PhSeCl}$ & $\mathrm{DMF}$ & 20 & 45 & $81 / 19$ \\
$5^{\mathrm{b}}$ & $\mathrm{PhSeCl}$ & $\mathrm{MeCN}$ & 32 & 40 & $88 / 12$ \\
$6^{\mathrm{b}}$ & $\mathrm{PhSeCl}$ & $\mathrm{DME}$ & 1 & 99 & - \\
$7^{\mathrm{b}}$ & $\mathrm{PhSeOTf}$ & $\mathrm{MeCN}$ & 43 & 29 & $87 / 13$ \\
$8^{\mathrm{b}}$ & $\mathrm{PhSeOTf}$ & $\mathrm{DMSO}^{\mathrm{DMSO}}$ & 73 & 10 & $87 / 13$ \\
$9^{\mathrm{b}}$ & $\mathrm{PhSeCl}$ & $\mathrm{bmimBF}_{4}$ & 24 & 20 & $81 / 19$ \\
\hline
\end{tabular}

${ }^{\mathrm{a}} \mathrm{NaN}_{3} 10$ eq.; ${ }^{\mathrm{b}} \mathrm{NaN}_{3} 5$ eq.; ${ }^{\mathrm{c}} \mathrm{NaN}_{3} 3$ eq. 
In each case the diastereoselectivity found is very similar ( $87: 13$, as determined by $\left.{ }^{1} \mathrm{H}-\mathrm{NMR}\right)$. Finally, the reaction was carried out in the ionic liquid 1-butyl-3-methylimidazolium tetrafluoroborate $\left(\mathrm{bmimBF}_{4}\right)$. The yield was poor and no improvement in diastereoselectivity was observed. Moreover, a 20\% yield of compounds 2a and 3a was obtained with high ratio (94:6) [12].

In order to establish the stereochemistry of the major product, the mixture of $4 \mathbf{a}$ and $5 \mathbf{a}$ was separated by column chromatography. The major product was reduced with triphenylphosphine and then treated with 1,1'-carbonyldiimidazole to give the cyclic urethane 9 (Scheme 3 ). The ${ }^{1} \mathrm{H}-\mathrm{NMR}$ spectrum showed that the coupling constants are small (see Experimental) confirming in this manner the structure proposed.

\section{Scheme 3}

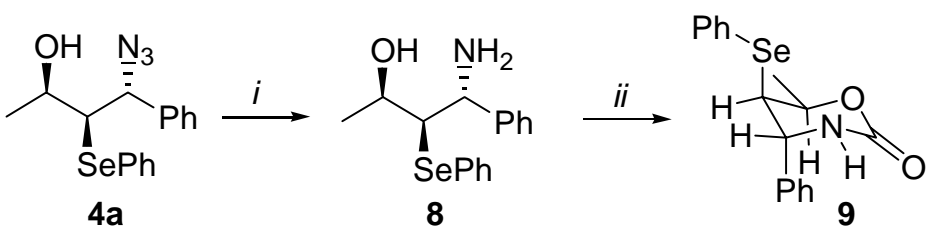

i: $\mathrm{PPh}_{3}, \mathrm{THF} / \mathrm{H}_{2} \mathrm{O}$, r.t.; ii: 1,1'-carbonyldiimidazole, $\mathrm{CH}_{2} \mathrm{Cl}_{2}$.

Using our best conditions (PhSeOTf 1eq., DMSO, $\mathrm{NaN}_{3} 5$ eq.), we carried out the azidoselenenylation on compounds $\mathbf{1 b}-\mathbf{g}$ (Table 3). Yields were not high and, usually, a considerable amount of starting material was recovered (30-45\%). No significant change in diastereoselectivity was found, being lower in the case of 1f; however, for substrates possessing an alkyl group as substituent on the $\mathrm{C}=\mathrm{C}$ double bond (1d,g), somewhat higher selectivities were observed. Protection of the hydroxy group in 1a as benzyl or TBDMS ether gave poor yields in the azido-selenenylation [13].

Table 3. Azido selenenylation of compounds 1a-g.

\begin{tabular}{ccccc}
\hline Entry & Compd. & $\mathbf{4 + 5}$ & $\mathbf{1}$ & $\mathbf{4 / 5}$ \\
\hline 1 & 1a & 73 & 10 & $87: 13$ \\
2 & 1b & 51 & 45 & $84: 16$ \\
3 & 1c & 64 & 30 & $83: 17$ \\
4 & 1d & 58 & 40 & $92: 8$ \\
5 & 1e & 33 & 33 & $87: 13$ \\
6 & 1f & 48 & 43 & $82: 18$ \\
7 & 1g & 57 & 35 & $93: 7$ \\
\hline
\end{tabular}

In order to prepare 2-phenylselenenyl-1,3-azido alcohols a different approach can be followed. We reasoned that using an allylic azide as starting material and water as nucleophile better yields could be reached. However, this approach suffers a limitation: allylic azides exist as an equilibrating mixture of regioisomers [14]. Such rearrangement can be suppressed in compounds in which the allylic azide is conjugated. To explore this notion we used compound 10 (Scheme 4). 


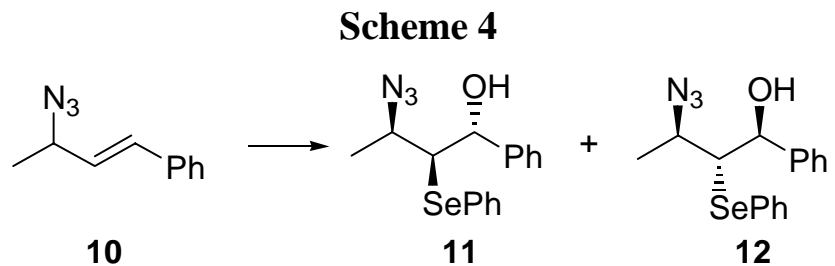

Under the usual conditions we obtained compounds 11 and $\mathbf{1 2}$ in 74\% yield and 90:10 ratio. Quenching the reaction after one hour we observed a lower yield (63\%); moreover, an even lower yield (55\%) was also obtained if water is added to a solution containing compound $\mathbf{1 0}$ and $\mathrm{PhSeCl}$ in $\mathrm{CH}_{3} \mathrm{CN}$. In each case the 11:12 ratio did not change. It is noteworthy that the 11:12 ratio is identical to the 2a:3a ratio, leading us to conclude that both the $\mathrm{OH}$ and $\mathrm{N}_{3}$ groups play a similar role.

Finally, we used compound 4a for further transformation. It is known that hydroxyselenides can be transformed into epoxides [15]. Treatment of $4 \mathbf{a}$ with 1.2 equiv. of $m$-chloroperbenzoic acid $/ \mathrm{K}_{2} \mathrm{CO}_{3}$ in methanol at $-10{ }^{\circ} \mathrm{C}$ did not afford the expected product. Rather, oxidative elimination via selenoxide syn-elimination took place to give compound 13 in $65 \%$ yield. However, when the reaction was carried out with 5 equiv. the cis-epoxide 14 [16] was obtained in 40\% yield. In the presence of an excess of MCPBA the reactive intermediate is the selenone. Itr would appear that because of the excellent leaving-group properties of the $\mathrm{PhSeO}_{2}$ group, the reaction indeed took place, but, probably due to steric hindrance, the yield was not high. The cis-stereochemistry was established by ${ }^{1} \mathrm{H}-\mathrm{NMR}\left(J_{\mathrm{H} 2 \mathrm{H} 3}=\right.$ $4.2 \mathrm{~Hz}$ ). Although the yield was not high this methodology allows the stereoselective synthesis of 1-azido-2,3-cis-epoxides, useful compounds for further manipulations.

\section{Scheme 5}

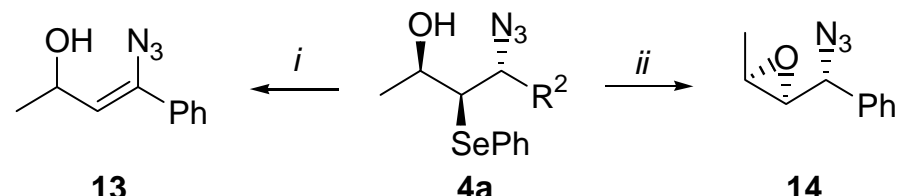

i: $\mathrm{MCPBA}$ (1.2 equiv.), $\mathrm{K}_{2} \mathrm{CO}_{3}$ (1.2 equiv.), $\mathrm{MeOH},-10{ }^{\circ} \mathrm{C}$; ii: $\mathrm{MCPBA}$ (5 equiv.), $\mathrm{K}_{2} \mathrm{CO}_{3}$ (5 equiv.), $\mathrm{MeOH},-10^{\circ} \mathrm{C}$.

\section{Conclusions}

We have presented a facile route for the synthesis of 2-phenylselenenyl-1,3-anti-diols and 2phenylselenenyl-1,3-anti-azido-alcohols with interesting diastereoselectivities and complete regioselectivity.

\section{Acknowledgments}

Financial support from the University of Palermo (funds for selected research topics) and Italian MIUR within the National Research Project "Non-aromatic heterocycles in stereocontrolled processes" is gratefully acknowledged. 


\section{Experimental}

\section{General}

${ }^{1} \mathrm{H}-\mathrm{NMR}$ and ${ }^{13} \mathrm{C}$ NMR spectra were recorded on a Bruker AC-E series $250 \mathrm{MHz}$ spectrometer as $\mathrm{CDCl}_{3}$. When a minor diastereoisomer was obtained in pure form, spectra and analytical data are reported. When minor diastereoisomers were obtained as mixtures, the distinguishable signals are reported. IR spectra were recorded on a Shimadzu model FTIR 8300 infrared spectrophotometer using $\mathrm{NaCl}$ cells. Flash chromatography was carried out using Macherey-Nagel silica gel (0.04-0.063 mm). Light petroleum refers to the fraction boiling in the range $40-60{ }^{\circ} \mathrm{C}$. Compounds 1a and 1e were prepared by sodium borohydride reduction of the corresponding commercially available ketones; compound $\mathbf{1 b}$ by reaction of cinnamaldehyde with $i$-propylmagnesium chloride; compounds $\mathbf{1 c}$ and $\mathbf{1 g}$ by sodium borohydride reduction of the corresponding ketone obtained by Wittig reaction; compound 1d by reaction of trans-crotonaldehyde with methylmagnesium chloride; compound $\mathbf{1 f}$ by reaction of cinnamaldehyde with lithium enolate of ethyl acetate and compound 8 by reaction with $\mathrm{NaN}_{3}$ on the acetyl derivative of compound $\mathbf{1 a}$ in the presence of $\mathrm{Pd}\left(\mathrm{PPh}_{3}\right)_{4}$ [17]. All compounds showed spectroscopic and analytical data in agreement with their structures.

\section{General procedure for hydroxy-selenenylation reactions:}

To a stirred solution of compounds $1 \mathbf{1 a}-\mathbf{f}$ in acetonitrile $(2 \mathrm{~mL}$ per mmol) and water (33 equiv.) a solution of $\mathrm{PhSeCl}$ ( 1 equiv.) in acetonitrile ( $1 \mathrm{~mL}$ per mmole) was added via cannula. After 3 minutes the reaction was quenched by addition of a saturated aqueous solution of $\mathrm{NaHCO}_{3}$ and the mixture was partitioned between ethyl acetate and water. The combined organic phases were washed with brine and dried. The crude product was purified by flash chromatography.

( \pm )-(1RS, 2SR, 3RS)-1-phenyl-2-phenylselenenyl-butan-1,3-diol (2a): Mixture with 3a. From light petroleum/diethyl ether $1: 1$; white solid, mp $52-53{ }^{\circ} \mathrm{C}$; IR (nujol) v: $3350,1570,1490,1470 \mathrm{~cm}^{-1}$; ${ }^{1} \mathrm{H}-\mathrm{NMR} \delta: 1.31\left(\mathrm{~d}, J=5.7 \mathrm{~Hz}, 3 \mathrm{H}, \mathrm{CH}_{3}\right), 1.36\left(\mathrm{~d}, J=6.0 \mathrm{~Hz}, 3 \mathrm{H}, \mathrm{CH}_{3}, 3 \mathrm{a}\right), 3.35$ (dd, $J=4.6$ and $1.6 \mathrm{~Hz}$,

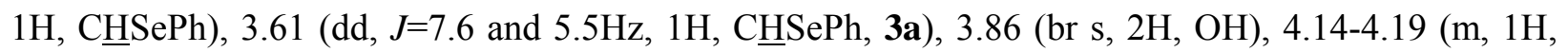

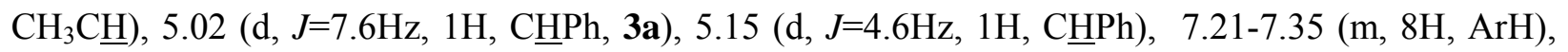
7.49-7.56 (m, 2H, ArH); ${ }^{13} \mathrm{C}-\mathrm{NMR} \delta: 21.9,61.1,66.8,76.7,126.0,127.5,128.1,128.3,129.1,134.4$, 141.9; Anal. Calcd. for $\mathrm{C}_{16} \mathrm{H}_{18} \mathrm{O}_{2}$ Se: C, 59.82; H, 5.65. Found: C, 60.39; H, 5.69.

( \pm )-(1RS, 2SR, 3RS)-4-methyl-1-phenyl-2-phenylselenenyl-pentan-1,3-diol (2b): Mixture with $3 \mathbf{b}$. From light petroleum/diethyl ether 3:1; oil; IR (liquid film) v: $3300,1455 \mathrm{~cm}^{-1} ;{ }^{1} \mathrm{H}-\mathrm{NMR} \delta: 0.68$ (d, $\left.J=6.6 \mathrm{~Hz}, 3 \mathrm{H}, \mathrm{CH}_{3}\right), 0.82$ (d, $\left.J=6.7 \mathrm{~Hz}, 3 \mathrm{H}, \mathrm{CH}_{3}, 3 \mathbf{b}\right), 0.93$ (d, $\left.J=6.7 \mathrm{~Hz}, 3 \mathrm{H}, \mathrm{CH}_{3}, 3 \mathbf{b}\right), 0.99$ (d, $J=6.7$, $\left.3 \mathrm{H}, \mathrm{CH}_{3}\right), 2.05-2.21\left(\mathrm{~m}, 1 \mathrm{H}, \mathrm{CHMe}_{2}\right), 3.44(\mathrm{dd}, J=9.2$ and $2.5 \mathrm{~Hz}, 1 \mathrm{H}, \mathrm{C} \underline{\mathrm{HSePh}}), 3.56(\mathrm{~d}, J=3.3 \mathrm{~Hz}$,

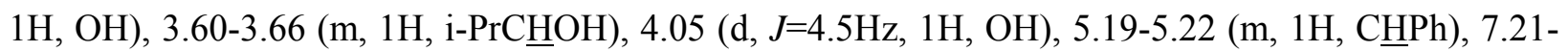
$7.44(\mathrm{~m}, 8 \mathrm{H}, \mathrm{ArH}), 7.65-7.69$ (m, 2H, ArH) $;{ }^{13} \mathrm{C}-\mathrm{NMR} \delta: 18.7,18.9,32.2,58.1,75.9,76.4,125.6$, 127.3, 127.7, 128.2, 129.2, 134.8, 141.3; Anal. Calcd. for $\mathrm{C}_{18} \mathrm{H}_{22} \mathrm{O}_{2} \mathrm{Se}$ : C, 61.89; H, 6.35. Found: C, $62.57 ; \mathrm{H}, 6.39$. 
( \pm )-(1RS, 2SR, 3RS)-1-p-chlorophenyl-2-phenylselenenyl-butan-1,3-diol (2c): Mixture with 3c. From light petroleum/diethyl ether 4:1; oil; IR (liquid film) v: 3350, 1590, 1575, 1485, $1470 \mathrm{~cm}^{-1} ;{ }^{1} \mathrm{H}-\mathrm{NMR}$ $\delta: 1.31\left(\mathrm{~d}, J=6.3 \mathrm{~Hz}, 3 \mathrm{H}, \mathrm{CH}_{3}\right), 1.36\left(\mathrm{~d}, J=6.2 \mathrm{~Hz}, 3 \mathrm{H}, \mathrm{CH}_{3}, 3 \mathrm{c}\right), 3.16(\mathrm{dd}, J=7.5$ and $1.5 \mathrm{~Hz}, 1 \mathrm{H}$,

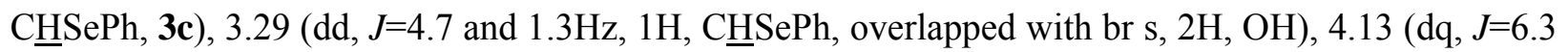
and $\left.1.3 \mathrm{~Hz}, 1 \mathrm{H}, \mathrm{CH}_{3} \mathrm{C} \underline{\mathrm{H}}\right), 5.08(\mathrm{~d}, J=4.7 \mathrm{~Hz}, 1 \mathrm{H}, \mathrm{C} \underline{\mathrm{HAr}}), 7.15-7.30(\mathrm{~m}, 7 \mathrm{H}, \mathrm{ArH}), 7.44-7.51$ (m, 2H, ArH); ${ }^{13} \mathrm{C}-\mathrm{NMR} \delta: 22.0,61.1,66.9,76.1,127.5,127.8,128.5,129.2,134.5,140.3$; Anal. Calcd. for $\mathrm{C}_{16} \mathrm{H}_{17} \mathrm{ClO}_{2} \mathrm{Se}: \mathrm{C}, 54.02 ; \mathrm{H}, 4.82$. Found: C, 54.40; H, 4.80.

( \pm )-(1RS, 3RS)-3-phenylselenenyl-pentan-1,3-diol (2d): Mixture with 3d. From light petroleum/diethyl ether 4:1; white solid, mp 52-53 ${ }^{\circ} \mathrm{C}$; IR (nujol) v: 3300, 1570, $1450 \mathrm{~cm}^{-1} ;{ }^{1} \mathrm{H}-\mathrm{NMR} \delta: 1.36(\mathrm{~d}$, $\left.J=6.5 \mathrm{~Hz}, 3 \mathrm{H}, \underline{\mathrm{CH}}_{3}\right), 1.40\left(\mathrm{~d}, J=6.3 \mathrm{~Hz}, 3 \mathrm{H}, \mathrm{CH}_{3}\right), 3.15(\mathrm{dd}, J=5.0$ and $2.7 \mathrm{~Hz}, 1 \mathrm{H}, \mathrm{C} \underline{\mathrm{HSePh}}), 3.21$ (dd, $J=7.0$ and $7.0 \mathrm{~Hz}, 1 \mathrm{H}, \mathrm{C} \underline{\mathrm{HS}} \mathrm{Ph}, 3 \mathrm{~d}$ ), 3.57 (br s, $2 \mathrm{H}, \mathrm{OH}), 4.18$ (dq, $J=6.5$ and $5.0 \mathrm{~Hz}, 1 \mathrm{H}, \mathrm{C} \underline{\mathrm{HOH}}$ ), 4.33 (dq, $J=6.3$ and $2.7 \mathrm{~Hz}, 1 \mathrm{H}, \mathrm{C} \underline{\mathrm{HOH}}$ ), 7.25-7.30 (m, 3H, ArH), 7.55-7.62 (m, 2H, ArH); ${ }^{13} \mathrm{C}-\mathrm{NMR} \delta$ : 21.5, 62.2, 67.1, 69.8, 127.4, 129.1, 129.5, 134.0; Anal. Calcd. for $\mathrm{C}_{11} \mathrm{H}_{16} \mathrm{O}_{2} \mathrm{Se}$ : C, 50.97; H, 6.22. Found: C, 51.10; 6.30.

( \pm )-(1RS, 3RS)-1,3-diphenyl-2-phenylselenenyl-propan-1,3-diol (2e): From light petroleum/diethyl ether 4:1; white solid, mp 137-138 ${ }^{\circ} \mathrm{C}$; IR (nujol) v: 3400, 1596, 1570, 1490, 1470, 1445, $1430 \mathrm{~cm}^{-1}$; ${ }^{1} \mathrm{H}-\mathrm{NMR} \delta$ : $3.56(\mathrm{dd}, J=3.9$ and $1.7 \mathrm{~Hz}, 1 \mathrm{H}, \mathrm{C} \underline{\mathrm{HS}} \mathrm{SePh}$ overlapped with br s, $1 \mathrm{H}, \mathrm{OH}$ ), 3.63 (br s, $1 \mathrm{H}$, $\mathrm{OH}), 5.13(\mathrm{~d}, J=1.7 \mathrm{~Hz}, 1 \mathrm{H}, \mathrm{C} \underline{\mathrm{HOH}}), 5.20(\mathrm{~d}, J=3.9 \mathrm{~Hz}, 1 \mathrm{H}, \underline{\mathrm{CHOH}}), 7.10-7.40(\mathrm{~m}, 15 \mathrm{H}, \operatorname{ArH})$; ${ }^{13} \mathrm{C}-\mathrm{NMR} \delta$ : 63.4, 71.5, 76.5, 125.7, 125.8, 127.2, 127.5, 127.7, 127.9, 128.5, 128.9, 134.4, 134.5, 141.3, 142.0; Anal. Calcd. for $\mathrm{C}_{21} \mathrm{H}_{20} \mathrm{O}_{2} \mathrm{Se}$ : C, 65.80; H, 5.26. Found: 66.60; H, 5.31.

\section{General procedure for azido-selenenylation reactions:}

To a stirred solution of $\mathrm{PhSeCl}$ ( 1 equiv.), $\mathrm{NaN}_{3}$ (5 equiv.) and $\mathrm{AgOTf}$ (1 equiv.) in DMSO (74 mL per $\mathrm{mmol}$ of $\mathrm{PhSeCl}$ ) a solution of compounds 1a-f in DMSO (4.4 $\mathrm{mL}$ per mmol) was added via cannula. After 24 hours the reaction was quenched by addition of water and the mixture was portioned between diethyl ether and water. The combined organic phases were washed with brine and dried. The crude product was purified by flash chromatography.

( \pm )-(2RS, 3RS, 4RS)-4-azido-4-phenyl-3-phenylselenenyl-butan-2-ol (4a): From light petroleum/diethyl ether 9:1; white solid, mp 62-63 ${ }^{\circ} \mathrm{C}$; IR (nujol) v: 3270, $2090 \mathrm{~cm}^{-1} ;{ }^{1} \mathrm{H}-\mathrm{NMR} \delta: 1.43(\mathrm{~d}, \mathrm{~J}=6.3 \mathrm{~Hz}, 3 \mathrm{H}$, $\mathrm{CH}_{3}$ ), 2.51 (br s, $\left.1 \mathrm{H}, \mathrm{OH}\right), 3.25$ (dd, $J=6.1$ and $\left.1.4 \mathrm{~Hz}, 1 \mathrm{H}, \mathrm{C} \underline{\mathrm{HSePh}}\right), 4.31$ (dq, $J=6.3$ and $1.4 \mathrm{~Hz}, 1 \mathrm{H}$, $\mathrm{CH}_{3} \mathrm{C} \underline{\mathrm{H}}$ ), 4.93 (d, J=6.1Hz, 1H, CㅌPh), 7.16-7.37 (m, 10H, ArH); ${ }^{13} \mathrm{C}-\mathrm{NMR} \delta: 22.4,60.8,66.2,68.9$, 127.5, 127.7, 128.4, 128.5, 129.9, 134.7, 137.8; Anal. Calcd. for $\mathrm{C}_{16} \mathrm{H}_{17} \mathrm{~N}_{3} \mathrm{OSe}$ : C, 55.49; H, 4.95. Found: C, 55.90; H, 5.05.

( \pm )-(2RS, 3SR, 4SR)-4-azido-4-phenyl-3-phenylselenenyl-butan-2-ol (5a): From light petroleum/diethyl ether 9:1; white solid, mp 87-88 ${ }^{\circ} \mathrm{C}$; IR (nujol) v: 3400, $2090 \mathrm{~cm}^{-1} ;{ }^{1} \mathrm{H}-\mathrm{NMR} \delta: 1.31 .(\mathrm{d}, \mathrm{J}=6.3 \mathrm{~Hz}, 3 \mathrm{H}$, $\left.\mathrm{CH}_{3}\right), 2.40$ (br s, $\left.1 \mathrm{H}, \mathrm{OH}\right), 3.53$ (dd, $J=9.7$ and $\left.4.5 \mathrm{~Hz}, 1 \mathrm{H}, \mathrm{C} \underline{\mathrm{HSePh}}\right), 4.10-4.20\left(\mathrm{~m}, 1 \mathrm{H}, \mathrm{CH}_{3} \mathrm{C} \underline{\mathrm{H}}\right), 4.76$ $(\mathrm{d}, J=9.7 \mathrm{~Hz}, 1 \mathrm{H}, \mathrm{C} \underline{\mathrm{HPh}}), 7.05-7.29(\mathrm{~m}, 10 \mathrm{H}, \mathrm{ArH}) ;{ }^{13} \mathrm{C}-\mathrm{NMR} \delta: 20.6,62.8,66.9,68.3,127.7,127.0$, 
128.4, 128.6, 128.9, 129.0, 134.6, 137.2; Anal. Calcd. for $\mathrm{C}_{16} \mathrm{H}_{17} \mathrm{~N}_{3} \mathrm{OSe}$ C, 55.49; H, 4.95. Found: C, $56.09 ; \mathrm{H}, 5.06$.

( \pm )-(2RS, 3RS, 4RS)-5-azido-2-methyl-5-phenyl-4-phenylselenenyl-pentan-3-ol (4b): Mixture with 5b. From light petroleum/diethyl ether 9:1; white solid, mp 68-71 ${ }^{\circ} \mathrm{C}$; IR (nujol) v: $3500,2090,1575 \mathrm{~cm}^{-1}$; ${ }^{1} \mathrm{H}-\mathrm{NMR} \delta: 0.81\left(\mathrm{~d}, J=6.6 \mathrm{~Hz}, 3 \mathrm{H}, \mathrm{CH}_{3}\right), 0.84\left(\mathrm{~d}, J=6.7 \mathrm{~Hz}, 3 \mathrm{H}, \mathrm{CH}_{3}, 5 \mathbf{b}\right), 1.04$ (d, $J=6.7 \mathrm{~Hz}, 3 \mathrm{H}, \mathrm{CH}_{3}$, 5b), 1.14 (d, J=6.6Hz, 3H, CH 3 ), 2.18-23 (m, 1H, $\underline{H M e}_{2}$ ), 2.70 (br s, 1H,OH), 3.56 (dd, J=7.2 and $0.8 \mathrm{~Hz}, 1 \mathrm{H}, \mathrm{C} \underline{\mathrm{HSePh}}), 3.64-3.68(\mathrm{~m}, 1 \mathrm{H}, \mathrm{CHOH}), 5.03(\mathrm{~d}, \mathrm{~J}=7.2 \mathrm{~Hz}, 1 \mathrm{H}, \mathrm{C} \underline{\mathrm{HPh}}), 5.25(\mathrm{~d}, J=7.4 \mathrm{~Hz}, 1 \mathrm{H}$, C바, 5b), 7.23-7.50 (m, 10H, ArH $) ;{ }^{13} \mathrm{C}-\mathrm{NMR} \delta: 18.9,19.1,32.2,57.2,69.1,75.6,127.4,127.7$, 128.2, 128.3, 128.5, 128.9, 135.0, 138.0; Anal. Calcd. for $\mathrm{C}_{18} \mathrm{H}_{21} \mathrm{~N}_{3} \mathrm{OSe}$ : C, 57.75; H, 5.65. Found: C, $58.23 ; \mathrm{H}, 5.80$.

( \pm )-(2RS, 3RS, 4RS)-4-azido-4-p-chlorophenyl-3-phenylselenenyl-butan-2-ol (4c): From light petroleum/diethyl ether 3:1; white solid, mp 54-55 ${ }^{\circ} \mathrm{C}$; IR (nujol) v: 3500, $2090 \mathrm{~cm}^{-1}$; ${ }^{1} \mathrm{H}-\mathrm{NMR}$ $\delta: 1.47\left(\mathrm{~d}, J=6.2 \mathrm{~Hz}, 3 \mathrm{H}, \mathrm{CH}_{3}\right), 2.49$ (br s, $\left.1 \mathrm{H}, \mathrm{OH}\right), 3.17$ (dd, $J=8.5$ and $\left.1.3 \mathrm{~Hz}, 1 \mathrm{H}, \mathrm{C} \underline{\mathrm{HSePh}}\right), 4.33$ (dq, $J=6.2$ and $1.3 \mathrm{~Hz}, 1 \mathrm{H}, \mathrm{CH}_{3} \mathrm{C} \underline{\mathrm{H}}$ ), 4.90 (d, $J=8.5 \mathrm{~Hz}, 1 \mathrm{H}, \mathrm{C} \underline{\mathrm{HAr}}$ ), 7.10-7.28 (m, 9H, ArH); ${ }^{13} \mathrm{C}-\mathrm{NMR}$ $\delta: 22.6,60.7,88.2,68.2,127.7,128.6,129.0,129.1,134.6,136.4$; Anal. Calcd. for $\mathrm{C}_{16} \mathrm{H}_{16} \mathrm{ClN}_{3} \mathrm{OSe}$ : C, 50.47; H, 4.24. Found: C, 51.01; H, 4.34.

( \pm$)-(2 R S, 3 R S, 4 R S)-4-a z i d o-3-p h e n y l s e l e n e n y l-p e n t a n-2-o l$ (4d): Mixture with 5d. From light petroleum/diethyl ether 7:1; oil; IR (liquid film) v: 3430, 2100, $1575 \mathrm{~cm}^{-1}$; ${ }^{1} \mathrm{H}-\mathrm{NMR} \delta: 1.30$ (d, $\left.J=6.4 \mathrm{~Hz}, 3 \mathrm{H}, \mathrm{CH}_{3}, 5 \mathrm{~d}\right), 1.41$ (d, $\left.J=6.3 \mathrm{~Hz}, 3 \mathrm{H}, \mathrm{CH}_{3}\right), 1.47$ (d, J=6.8Hz, 3H, $\left.\mathrm{CH}_{3}\right), 2.68$ (br s, $\left.1 \mathrm{H}, \mathrm{OH}\right)$, $3.04(\mathrm{dd}, J=6.5$ and $2.5 \mathrm{~Hz}, 1 \mathrm{H}, \mathrm{C} \underline{H} \mathrm{SePh}), 3.26(\mathrm{dd}, J=7.0$ and $5.8 \mathrm{~Hz}, 1 \mathrm{H}, \mathrm{C} \underline{\mathrm{HS}} \mathrm{Ph}, 5 \mathrm{~d}), 3.91$ (dq, $J=6.8$ and $\left.6.5 \mathrm{~Hz}, 1 \mathrm{H}, \mathrm{CHCH}_{3}\right), 4.21\left(\mathrm{dq}, J=6.3\right.$ and $\left.2.5 \mathrm{~Hz}, 1 \mathrm{H}, \underline{\mathrm{HCH}}_{3}\right), 7.27-7.32(\mathrm{~m}, 3 \mathrm{H}, \mathrm{ArH})$, 7.59-7.66 (m, 2H, ArH); ${ }^{13} \mathrm{C}-\mathrm{NMR} \delta: 18.2,22.1,60.3,61.6,66.6,127.5,129.1$, 129.6, 134.0; Anal. Calcd. for $\mathrm{C}_{11} \mathrm{H}_{15} \mathrm{~N}_{3} \mathrm{OSe}$ : C, 46.48; H, 5.32. Found: C, 46.90; H, 5.40.

( \pm )-(2RS, 3RS, 4RS)-3-azido-1,3-diphenyl-2-phenylselenenyl-propan-1-ol $\quad(4 \mathbf{e}):$ From light petroleum/diethyl ether 15:1; white solid, mp 77-78 ${ }^{\circ} \mathrm{C}$; IR (nujol) v: $3400,2100 \mathrm{~cm}^{-1} .{ }^{1} \mathrm{H}-\mathrm{NMR} \delta$ : 2.94 (br s, $1 \mathrm{H}, \mathrm{OH}), 3.38$ (dd, $J=7.8$ and $1.9 \mathrm{~Hz}, 1 \mathrm{H}, \mathrm{C} \underline{\mathrm{HSePh}}), 5.00$ (d, $J=7.8 \mathrm{~Hz}, 1 \mathrm{H}, \mathrm{C} \underline{\mathrm{HPh}}$ ), 5.33 (d, $J=1.9 \mathrm{~Hz}, 1 \mathrm{H}, \mathrm{C} \underline{\mathrm{HPh}}), 6.8-6.82$ (m, 2H, ArH), 7.11-7.17 (m, 1H, ArH), 7.23-7.37 (m, 10H, ArH); ${ }^{13} \mathrm{C}-$ NMR $\delta: 62.6,69.0,71.4,126.0,127.4,127.5,127.6,128.1,128.5,128.6,128.7,134.8,137.8,142.2$; Anal. Calcd. for $\mathrm{C}_{21} \mathrm{H}_{19} \mathrm{~N}_{3} \mathrm{OSe}$ C, 61.77; H, 4.69. Found: C, 62.10; H, 4.74.

( \pm )-(3RS, 4RS, 5RS)-5-azido-3-hydroxy-4-phenylselenenyl-5-phenyl-pentanoate ethyl ester (4f): From light petroleum/diethyl ether 3:1; white solid, mp 92-93 ${ }^{\circ} \mathrm{C}$; IR (nujol) v: 3430, 2100, $1575 \mathrm{~cm}^{-1}$; ${ }^{1} \mathrm{H}-\mathrm{NMR} \delta: 1.26\left(\mathrm{t}, J=7.1 \mathrm{~Hz}, 3 \mathrm{H}, \mathrm{OCH}_{2} \mathrm{CH}_{3}\right), 2.64(\mathrm{dd}, J=16.4$ and $4.0 \mathrm{~Hz}, 1 \mathrm{H}, \mathrm{EtOCOCHH}), 3.16$ (dd, $J=16.4$ and $9.3 \mathrm{~Hz}, 1 \mathrm{H}, \mathrm{EtOCOCH} \underline{\mathrm{H}}), 3.20(\mathrm{dd}, J=9.3$ and $1.2 \mathrm{~Hz}, 1 \mathrm{H}, \mathrm{C} \underline{\mathrm{HSePh}}), 3.37$ (br s, $1 \mathrm{H}$, $\mathrm{OH}), 4.16$ (q, $\left.J=7.1 \mathrm{~Hz}, 2 \mathrm{H}, \mathrm{OC}_{2} \mathrm{CH}_{3}\right), 4.64-4.69$ (m, $\left.1 \mathrm{H}, \mathrm{CH}_{2} \mathrm{C} \underline{\mathrm{HOH}}\right), 4.94$ (d, J=9.3Hz, $1 \mathrm{H}, \mathrm{C} \underline{\mathrm{HPh}}$ ), 7.07-7.32 (m, 10H, ArH); ${ }^{13} \mathrm{C}-\mathrm{NMR} \delta: 14.1,40.7,58.6,60.8,66.7,68.5,127.5,127.9,128.3,128.8$, 134.7, 137.6, 172.2; Anal. Calcd. for $\mathrm{C}_{19} \mathrm{H}_{21} \mathrm{~N}_{3} \mathrm{O}_{3}$ Se: C, 54.55; H, 5.06. Found: C, 54.99; H, 5.15. 
( \pm )-(2RS, 3RS, 4RS)-4-azido-5-phenyl-3-phenylselenenyl-pentan-2-ol (4g): From light petroleum/ diethyl ether 10:1; oil; IR (liquid film) v: 3330, $2100 \mathrm{~cm}^{-1} ;{ }^{1} \mathrm{H}-\mathrm{NMR} \delta: 1.43$ (d, J=6.3Hz, $3 \mathrm{H}, \mathrm{CH}_{3}$ ), 2.59 (br s, $1 \mathrm{H}, \mathrm{OH}), 2.92$ ( dd, $J=13.8$ and $8.8 \mathrm{~Hz}, 1 \mathrm{H}, \mathrm{PhCHH}$ ), 3.11 (dd, $J=5.3$ and 1.8Hz, $1 \mathrm{H}$, CㅂSePh), 3.22 (dd, $J=13.8$ and $5.4 \mathrm{~Hz}, 1 \mathrm{H}, \mathrm{PhCH} \underline{\mathrm{H}}), 3.97-4.05\left(\mathrm{~m}, 1 \mathrm{H}, \underline{\mathrm{CHN}}_{3}\right), 4.34$ (dt, $J=6.3$ and $1.8 \mathrm{~Hz}, 1 \mathrm{H}, \mathrm{C} \underline{\mathrm{HOH}}), 7.11-7.15(\mathrm{~m}, 2 \mathrm{H}, \mathrm{ArH}), 7.25-7.32(\mathrm{~m}, 6 \mathrm{H}, \mathrm{ArH}), 7.46-7.50(\mathrm{~m}, 2 \mathrm{H}, \operatorname{ArH})$; ${ }^{13} \mathrm{C}-\mathrm{NMR}$ 8: 22.4, 39.7, 58.4, 66.4, 67.7, 126.9, 127.5, 128.7, 129.1, 129.3, 133.9, 137.1; Anal. Calcd. for $\mathrm{C}_{17} \mathrm{H}_{19} \mathrm{~N}_{3} \mathrm{OSe}$ : C, 56.67; H, 5.32. Found: C, 56.99; H, 5.35.

( \pm )-(2RS, 3SR, 4SR)-4-azido-5-phenyl-3-phenylselenenyl-pentan-2-ol (5g): From light petroleum/ diethyl ether 10:1; oil. IR (liquid film) v: $3370,2100 \mathrm{~cm}^{-1} ;{ }^{1} \mathrm{H}-\mathrm{NMR} \delta: 1.35$ (d, J=6.3Hz, $3 \mathrm{H}, \mathrm{CH}_{3}$ ), 2.29 (br s, 1H, OH), 2.75 ( dd, $J=13.9$ and $9.9 \mathrm{~Hz}, 1 \mathrm{H}, \mathrm{PhCHH}), 3.36(\mathrm{dd}, J=7.2$ and $5.3 \mathrm{~Hz}, 1 \mathrm{H}$,

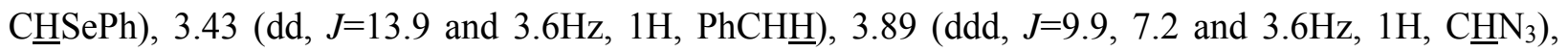
$4.34(\mathrm{dt}, J=6.3$ and $5.3 \mathrm{~Hz}, 1 \mathrm{H}, \mathrm{CHOH}), 7.20-7.35$ (m, 8H, ArH), 7.59-7.63 (m, 2H, ArH); ${ }^{13} \mathrm{C}-\mathrm{NMR}$ $\delta: 21.4,39.8,60.8,66.5,67.6,126.9,128.0,128.7,129.3,129.5,134.1,137.6$; Anal. Calcd. for $\mathrm{C}_{17} \mathrm{H}_{19} \mathrm{~N}_{3} \mathrm{OSe}: \mathrm{C}, 56.67 ; \mathrm{H}$, 5.32. Found: C, 57.05; H, 5.41.

(士)-(4RS, 5RS, 6RS)-6-methyl-4-phenyl-5-phenylselenenyl-1,3-oxazolidin-2-one (9)

To a solution of compound 4a (100 mg, $0.298 \mathrm{mmol})$ in dry THF (6 mL), $\mathrm{PPh}_{3}(84 \mathrm{mg}, 1.1 \mathrm{equiv}$.) was added. The solution was stirred at $40{ }^{\circ} \mathrm{C}$ overnight. After this time, water $(0.30 \mathrm{~mL})$ was added and the solution stirred at $50{ }^{\circ} \mathrm{C}$ for $3 \mathrm{~h}$. Methanol was then added and the solution evaporated under reduced pressure. The residue was dessiccated under vacuum in the presence of $\mathrm{P}_{2} \mathrm{O}_{5}$ for $5 \mathrm{~h}$. The white residue obtained was used without further purification in the next step. The residue (95 $\mathrm{mg}$ ) was dissolved in $\mathrm{CH}_{2} \mathrm{Cl}_{2}(2 \mathrm{~mL})$ at $0{ }^{\circ} \mathrm{C}$ then $1,1^{\prime}$ '-carbonyldiimidazole (58 mg, $\left.0.36 \mathrm{mmol}\right)$ was added. The solution was allowed to warm at room temperature then stirred for $48 \mathrm{~h}$. After this time the solvent was removed under reduced pressure and the residue purified by flash chromatography using $\mathrm{CH}_{2} \mathrm{Cl}_{2} /$ ethyl acetate 7:1 as eluent to afford compound 9 (34 mg, 34\%) as yellow solid, m.p. 175-177 ${ }^{\circ} \mathrm{C}$; IR (nujol) v: $3350,1720,1690,1455 \mathrm{~cm}^{-1}$; ${ }^{1} \mathrm{H}-\mathrm{NMR} \delta: 1.50$ (d, J=6.4 Hz, 3H, $\left.\mathrm{CH}_{3}\right), 3.44-3.46$ (m, 1H, C $\left.\underline{H} S e P h\right)$, $4.55\left(\mathrm{dt}, J=6.4\right.$ and $\left.2.5 \mathrm{~Hz}, 1 \mathrm{H}, \mathrm{CHCH}_{3}\right), 4.72(\mathrm{dd}, J=3.6 \mathrm{~Hz}, 1 \mathrm{H}, \mathrm{C} \underline{\mathrm{HPh}}), 5.81(\mathrm{~d}, J=3.6 \mathrm{~Hz}, 1 \mathrm{H}, \mathrm{NH})$, 7.12-7.16 (m, 2H, ArH), 7.27-7.37 (m, 6H, ArH), 7.52-7.56 (m, $2 \mathrm{H}, \operatorname{ArH}) ;{ }^{13} \mathrm{C}-\mathrm{NMR} \delta: 18.8,49.1$, $59.4,72.0,126.3,128.3,128.6,128.9,129.5,135.4,140.5,153.5$.

\section{Hydroxy-selenenylation reaction of compound $\mathbf{1 0}$}

To a stirred solution of compound $10(100 \mathrm{mg}, 0.58 \mathrm{mmol})$ in acetonitrile $(1 \mathrm{~mL})$ and water $(340$ $\mu \mathrm{L})$ a solution of $\mathrm{PhSeCl}(110 \mathrm{mg}, 1$ equiv.) in acetonitrile $(0.8 \mathrm{~mL})$ was added via cannula. After 3 minutes the reaction was quenched by addition of a saturated aqueous solution of $\mathrm{NaHCO}_{3}$ and the mixture was portioned between diethyl ether and water. The combined organic phases were washed with brine and dried. The crude product was purified by flash chromatography to give $( \pm)-(1 R S, 2 S R$, 3RS)-3-azido-1-phenyl-2-phenylselenenyl-butan-1-ol (11) as a mixture with 12. From light petroleum/diethyl ether 9:1; oil; IR (liquid film) v: 3430, 2100, $1575 \mathrm{~cm}^{-1} ;{ }^{1} \mathrm{H}-\mathrm{NMR} \delta: 1.44(\mathrm{~d}$, $\left.J=6.5 \mathrm{~Hz}, 3 \mathrm{H}, \mathrm{CH}_{3}, 12\right), 1.52$ (d, $\left.J=6.5 \mathrm{~Hz}, 3 \mathrm{H}, \mathrm{CH}_{3}\right), 2.95$ (d, $\left.J=3.1 \mathrm{~Hz}, 1 \mathrm{H}, \mathrm{OH}\right), 3.19$ (dd, $J=7.5$ and 
$1.6 \mathrm{~Hz}, 1 \mathrm{H}, \mathrm{C} \underline{\mathrm{HSePh}}), 3.51$ (dd, $J=6.1$ and $6.1 \mathrm{~Hz}, 1 \mathrm{H}, \mathrm{CHSePh}, 12), 3.80-3.85\left(\mathrm{~m}, 1 \mathrm{H}, \mathrm{CHN}_{3}, 12\right)$, $4.25\left(\mathrm{dq}, J=6.5\right.$ and $\left.1.6 \mathrm{~Hz}, 1 \mathrm{H}, \mathrm{CHN}_{3}\right), 4.92(\mathrm{dd}, J=7.5$ and $3.1 \mathrm{~Hz}, 1 \mathrm{H}, \mathrm{C} \underline{H P h}), 7.11-7.31(\mathrm{~m}, 10 \mathrm{H}$, ArH); ${ }^{13} \mathrm{C}-\mathrm{NMR} \delta: 19.1,56.5,60.7,76.4,126.8,127.5,127.8,128.2,128.8,129.0,134.6,141.6$.

\section{Oxidation of compound 4a}

To a solution of compound 4a $(100 \mathrm{mg}, 0.312 \mathrm{mmol})$ in methanol $(3 \mathrm{~mL})$ at $-10{ }^{\circ} \mathrm{C}$ was added $\mathrm{K}_{2} \mathrm{CO}_{3}(261 \mathrm{mg})$. After $10 \mathrm{~min} \mathrm{~m}$-chloroperbenzoic acid (269 mg, $1.56 \mathrm{mmol}$ ) was added. The reaction was quenched by adding water then the solution was partitioned between water and diethyl ether. The organic phase was washed with $\mathrm{Na}_{2} \mathrm{~S}_{2} \mathrm{O}_{3}$, dried and evaporated under reduced pressure. Purification by flash chromatography with light petroleum/diethyl ether 4/1 gave ( \pm )-(2SR, 3RS, 4RS)-4-azido-4phenyl-butan-2,3-oxirane (14) as an oil; IR (liquid film) v: 2090, $1620 \mathrm{~cm}^{-1} ;{ }^{1} \mathrm{H}-\mathrm{NMR} \delta: 1.42(\mathrm{~d}$, $\left.J=5.6 \mathrm{~Hz}, 3 \mathrm{H}, \mathrm{CH}_{3}\right), 3.10-3.19(\mathrm{~m}, 1 \mathrm{H}), 3.25(\mathrm{dd}, J=8.8$ and $4.2 \mathrm{~Hz}, 1 \mathrm{H}), 4.39(\mathrm{~d}, J=8.8 \mathrm{~Hz}, 1 \mathrm{H}), 7.32-$

7.45 (m, 5H, ArH); ${ }^{13} \mathrm{C}-\mathrm{NMR} \delta: 13.8,52.4,59.5,64.6,126.8,128.8,129.0,136.0$; Anal. Calcd. for $\mathrm{C}_{10} \mathrm{H}_{11} \mathrm{~N}_{3} \mathrm{O}: \mathrm{C}, 63.48 ; \mathrm{H}, 5.86$. Found: 64.10; H, 5.92.

(Z)-4-azido-4-phenyl-but-3-en-2-ol (13) was obtained following the same procedure but using 1.2 equivalents of $\mathrm{K}_{2} \mathrm{CO}_{3}$ and MCPBA: Yellow oil; IR (liquid film) v: 3420, 2100, $1638 \mathrm{~cm}^{-1}$; ${ }^{1} \mathrm{H}-\mathrm{NMR} \delta$ : $1.34\left(\mathrm{~d}, J=6.4 \mathrm{~Hz}, 3 \mathrm{H}, \mathrm{CH}_{3}\right), 2.00(\mathrm{br} \mathrm{s}, 1 \mathrm{H}, \mathrm{OH}), 4.85(\mathrm{dt}, J=7.8$ and $6.4 \mathrm{~Hz}, 1 \mathrm{H}), 5.22(\mathrm{~d}, J=7.8 \mathrm{~Hz}$, 1H), 7.36-7.45 (5H, ArH); Anal. Calcd. for $\mathrm{C}_{10} \mathrm{H}_{11} \mathrm{~N}_{3} \mathrm{O}$ : C, 63.48; H, 5.86. Found: C, 64.00; H, 5.90.

\section{References and Notes}

1. Rychnovsky, S. D. Oxo polyene macrolide antibiotics. Chem. Rev. 1995, 95, 2021-2040.

2. See for example: (a) Oishi, T.; Nakata, T. New aspects of stereoselective synthesis of 1,3-polyols. Synthesis 1990, 635-645; (b) Enders, D.; Hundertmark, T.; Lampe, C.; Jegelka, U.; Scharfbillig, I. Highly diastereo- and enantioselective synthesis of protected anti-1,3-diols. Eur. J. Org. Chem. 1998, 2839-2849 and references cited therein.

3. Toujas, J.-L.; Toupet, L.; Vaultier, M. Organometallic additions to $\beta$-substituted $N$-Boc- $\beta$ aminoaldehydes: a new synthesis of enantiomerically pure 1,3-disubstituted $N$-Boc-1,3aminoalcohols. Tetrahedron 2000, 56, 2665-2672 and references cited therein.

4. (a) Aprile, C.; Gruttadauria, M.; Amato, M. E.; D’Anna, F.; Lo Meo, P.; Riela, S.; Noto, R. Studies on the stereoselective selenolactonization, hydroxy and methoxy selenenylation of $\alpha$ - and $\beta$-hydroxy acids and esters. Synthesis of $\delta$ - and $\gamma$-lactones. Tetrahedron 2003, 59, 2241-2251; (b) Gruttadauria, M.; Aprile, C.; Lo Meo, P.; Riela, S.; Noto, R. Diastereoselective synthesis of substituted 2-phenyltetrahydropyrans as useful precursors of aryl C-glycosides via selenoetherification. Heterocycles 2004, 63, 681-690 and references cited therein.

5. Wirth, T., Ed. "Organoselenium Chemistry, Modern Developments in Organic Synthesis" Top. Curr. Chem. 2004, 208.

6. Only few examples are reported: (a) Cooper, M. A.; Ward, A. D. Hydroxyselenation of allylic alcohols. Tetrahedron Lett. 1995, 36, 2327-2330; (b) Haughan, A. F.; Knight, J. R.; Sweeney, J. B. Hydroxyselenation of acetoxycyclohex-2-ene. Tetrahedron Lett. 1994, 35, 1781-1784. 
7. (a) Tiecco, M.; Testaferri, L.; Temperini, A.; Bagnoli, L.; Marini, F.; Santi, C. Electrophilic azido selenenylation of alkenes. A simple synthetic route to racemic taxol side chain. Synthetic Commun. 1998, 28, 2167-2179; (b) Tiecco, M.; Testaferri, L.; Santi, C.; Tomassini, C.; Marini, F.; Bagnoli, L.; Temperini, A. Asymmetric azidoselenenylation of alkenes: a key step for the synthesis of enantiomerically enriched nitrogen-containing compounds. Angew. Chem. Int. Ed. 2003, 42, 3131-3133.

8. Hassner, A.; Amarasekara, A. S. Phenylselenium azide addition to alkenes. A new and stereospecific introduction of Se and N into organic molecules. Tetrahedron Lett. 1987, 28, 51855188.

9. Tingoli, M.; Tiecco, M.; Chianelli, D.; Balducci, R.; Temperini, A. Novel azidophenylselenenylation of double bonds. Evidences for a free radical process. J. Org. Chem. 1991, 56, 6809-6813

10. Ponthieux, S.; Outurquin, F.; Paulmier, C. Cross-aldol reaction between benzaldehyde and $\beta$ phenylselanyl enoxysilanes derived from phenylselanylmethylketones. Tetrahedron Lett. 1998, 39, 4017-4020.

11. Hori, T.; Sharpless, K. B. Synthetic applications of arylselenenic and arylseleninic acids. Conversion of olefins to allylic alcohols and epoxides. J. Org. Chem. 1978, 43, 1689-1696.

12. This reaction takes place because of the small amount of water contained in the $\mathrm{bmimBF}_{4}$ ionic liquid.

13. It is interesting to note that the diastereomeric ratio observed with the TBDMS derivative of compound 1a was still high (91:9).

14. (a) Padwa, A.; Sá, M. M. Intramolecular O-H insertion reaction of azido substituted diazoesters and its relevance to the mechanism of the allylic azide rearrangement. Tetrahedron Lett. 1997, 38, 5087-5090; (b) Arimoto, M.; Yamaguchi, H.; Fujita, E. Iodosylbenzene-trimethylsilyl azide-boron trifluoride etherate: a highly efficient system for direct synthesis of allyl azides from allylsilanes. Tetrahedron Lett. 1987, 28, 6289-6292.

15. (a) Toshimitsu, A.; Uemura, S. In Organoselenium Chemistry; Back, T. G., Ed.; Oxford University Press: Oxford, 1999; Chapter 13, p. 244; (b) Uemura, S.; Ohe, K.; Sugita, N. Oxidative conversion of $\beta$-hydroxyselenides to epoxides and ketones with meta-chloroperbenzoic acid. $J$. Chem. Soc., Chem. Commun. 1988, 111-112; (c) Krief, A.; Dumont, W.; Laboureur, J. L. Exploratory study on $\alpha$-metallo selenones. Original syntheses of oxaspiropentanones. Tetrahedron Lett. 1988, 29, 3265-3268.

16. Napolitano, E.; Fiaschi, R. Addition of iodine azide to allylic alcohols. Stereospecific synthesis of 3-azido-1,2-epoxides. Gazz. Chim. Ital. 1992, 122, 233-235.

17. Murahashi, S.-I.; Taniguchi, Y.; Imada, Y.; Tanigawa, Y. Palladium(0)-catalyzed azidation of allyl esters. Selective synthesis of allyl azides, primary allylamines, and related compounds. $J$. Org. Chem. 1989, 54, 3292-3303.

Sample availability: Available from the authors.

(C) 2005 by MDPI (http://www.mdpi.org). Reproduction is permitted for noncommercial purposes. 